\title{
Testing and Representation of Strain Rate Sensitive Mechanical Properties of Epoxy Resin
}

\author{
Qing Pan ${ }^{1, a}$, Weiyi Kong ${ }^{1, b}$, Shuguang $\mathrm{Li}^{2, \mathrm{c}}$, Elena Sitnikova ${ }^{2, d}$ \\ ${ }^{1}$ AECC, AECC Commercial Aircraft Engine Co., Ltd., Shanghai, China \\ ${ }^{2}$ Faculty of Engineering, University of Nottingham, Nottingham, NG7 2RD, UK \\ apanqingnuaa@163.com, bkongweiyiacae@163.com \\ 'Shuguang.Li@nottingham.ac.uk, dElena.Sitnikova@nottingham.ac.uk
}

Keywords: Epoxy resin, Strain rate sensitivity, Mechanical properties representation.

\begin{abstract}
To evaluate the strain rate sensitive mechanical properties of epoxy resin under high strain rate loading, the static and dynamic compression properties of PRIME ${ }^{\mathrm{TM}}$ epoxy resin was tested. To identify an appropriate method for assigning rate sensitivity to the material properties of the epoxy resin, three empirical models which suggest logarithmic or exponential dependency of the elastic and strength properties on the strain rate were applied to fit the experimental data. The capability of the three models were compared and discussed.
\end{abstract}

\section{Introduction}

Impact resistance is a significant mechanical property which should be investigated when applying carbon fiber reinforced polymer composites to aircraft engine. When considering constitutive behavior of carbon fiber reinforced composite material under dynamic loading, it is necessary to take into account the strain rate sensitivity of these materials. The rate dependent behavior of composites, which are generally strain rate sensitive materials, has been investigated in a number of studies. However, despite there being numerous publications, there is still a lack of clarity as to how the rate dependent composite behavior should be represented, as different studies report different results.

The Split Hopkinson pressure bar (SHPB) [1] is a well-known characterization tool for the mechanical response of materials loaded at high strain rates. Generally, the SHPB system was used to characterize the compressive properties of materials. In addition to the compression version, there are other modified versions with loading mechanisms for tension, torsion and their combinations [오류! 책갈피가 정의되어 있지 않습니다., 2].

Hufenbach et al. [3, 4], presented a rheological model to identify the strain rate deformation and failure behavior of textile reinforced polypropylene. Mohotti et al. [5] proposed a strain rate dependent constitutive material model to predict the high strain rate behavior of polyurea. Their model was based on the well-known nine parameter Mooney-Rivlin material model.

Daniel et al. [6, 7] proposed a strain rate dependent failure criteria for unidirectional carbon/epoxy material under multi-axial loading. From the tests, they found both modulus and strength were strain rate sensitive.

Cowper and Symonds [8] proposed an empirical model (known as the Cowper-Symonds model) to describe the strain rate hardening of metals. Yen [9] proposed a two parameters scaled model. In their model, the stiffness and strength are scaled by a ratio which is related to current strain rate. A reference strain rate has to be defined in this model, strain rates smaller than this value were treated as static. Therefore, the strain rate of static test was selected as reference strain rate.

From the above review, it is easy to see that strain rate sensitivity of composites is a complicated matter and it is therefore difficult to say which model is the best. For a specific material, experiments are required to determine the suitability of the model employed. The accuracies of different models can be judged by comparing the simulation results and the experimental results.

For the carbon fiber reinforced polymer composite materials, some of the published research [10] 
indicates that the rate-sensitive behavior exhibited mainly in the resin, whereas the carbon fiber can be treated as a rate-independent.

The presented work were carried out to evaluate the stain rate sensitive mechanical properties of epoxy resin and propose an appropriate models for representing the strain rate-depend material properties of the epoxy resin matrix in a composite material unit cell model.

\section{Mechanical Testing of the Epoxy Resin}

To determine the rate sensitivity of the Epoxy resin, appropriate experiments have been carried out with pure resin samples under compression at quasi-static and high strain rate loading.

The pure resin samples were produced with the PRIME ${ }^{\mathrm{TM}}$ epoxy resin system supplied by Gurit. The PRIME $^{\mathrm{TM}}$ 20LV resin and PRIME ${ }^{\mathrm{TM}}$ slow hardener were mixed in a weight ratio of 100:26 and degassed before being poured into aluminum tubes with inner diameter of $19 \mathrm{~mm}$, as shown in Fig. 1 (a). After being cured in an oven at $65^{\circ} \mathrm{C}$ for 7 hours, the resin rods as shown in Fig. 1 (b) were extracted from the tubes, and cut into $20 \mathrm{~mm}$ and $8 \mathrm{~mm}$ thick disks as the test specimens, respectively for different types of tests.

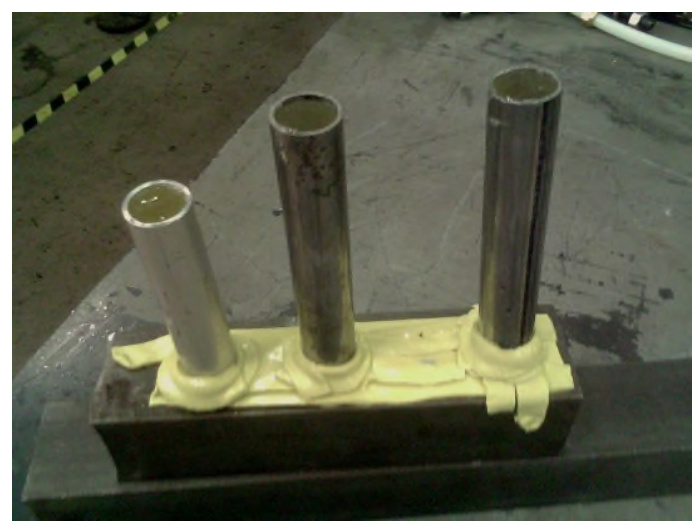

(a) Aluminum tubes filled with the resin

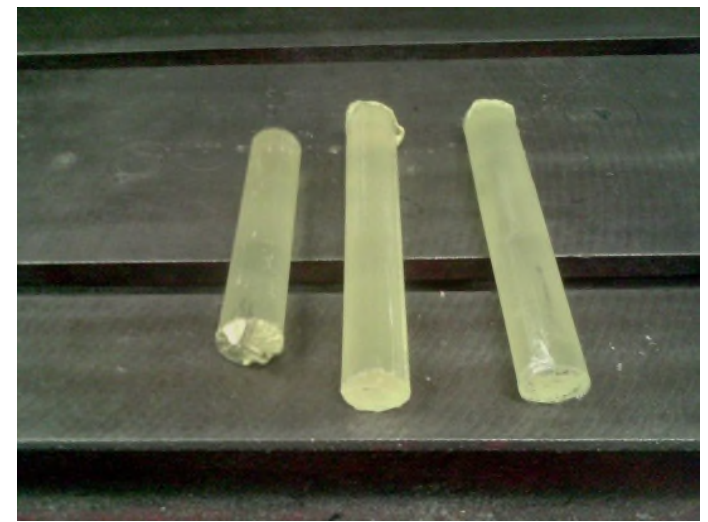

(b) Cured resin samples

Fig. 1 Preparation of resin samples.

Six specimens of $20 \mathrm{~mm}$ thickness were tested under quasi-static compression at a strain rate of $0.0004 \mathrm{~s}^{-1}$. The compressive load was applied by a 50kN Instron machine. The axial force was measured by the load cell located in the upper cross head. The displacement measurements were taken by two LVDT probes, positioned on either side of the specimen. These devices were interfaced with a PC. The measured force and displacement data were processed to give the compressive behavior of the resin in terms of stress-strain curves for the quasi-static stress state. The sample was assumed to be a perfect cylinder. To ensure good contact between the upper and the lower surfaces of the sample and the loading platens of the testing machine, the two ends of the sample were polished with a sample polishing machine and 240 grit sand paper. RS $^{\circledR}$ High Temperature Grease was applied on the specimen to reduce the fiction between the sample and the loading platens of the testing machine.

The Young's moduli and strengths determined in all six experiments are summarized in

Table 1. The experimental data are consistent, as there is little variation within stiffness and strength values measured in all the tests.

To investigate the rate-dependent behavior of the resin at high strain rates, the resin samples were tested with standard Split Hopkinson Pressure Bar (SHPB) set-up [11]. In this case, the $8 \mathrm{~mm}$ thick disk specimens were used. The specimens were polished using a sample polishing machine and 240 grit sand paper. RS $^{\circledR}$ High Temperature Grease was applied to reduce the fiction between the specimens and the pressure bars in a similar way as in the quasi-static tests.

A total of sixteen $8 \mathrm{~mm}$ thickness samples were tested at strain rates in the range from $763 \mathrm{~s}^{-1}$ to $2543 \mathrm{~s}^{-1}$. The measured Young's modulus and strength values are summarized in 
Table 1 along with the quasi-static test data.

Table 1. Compressive Young's modulus and strengths at different strain rates.

\begin{tabular}{cccc}
\hline NO. & $\dot{\varepsilon}\left(\mathrm{s}^{-1}\right)$ & $E(\mathrm{GPa})$ & $S_{c}(\mathrm{MPa})$ \\
\hline Static & $4 \times 10-4$ & 1.85 & 71.39 \\
1 & 763 & 2.15 & 99.54 \\
2 & 1046 & 2.52 & 142.48 \\
3 & 1158 & 2.15 & 159.38 \\
4 & 1180 & 2.90 & 192.34 \\
5 & 1326 & 2.38 & 181.76 \\
6 & 1552 & 2.61 & 218.92 \\
7 & 1698 & 1.84 & 190.65 \\
8 & 1843 & 2.45 & 219.80 \\
9 & 1884 & 2.26 & 188.96 \\
10 & 1920 & 2.28 & 222.71 \\
11 & 1990 & 2.82 & 230.43 \\
12 & 2033 & 2.09 & 219.11 \\
13 & 2041 & 2.65 & 227.77 \\
14 & 2451 & 2.86 & 210.45 \\
15 & 2543 & 2.48 & 225.11 \\
16 & 2920 & 2.79 & 248.02 \\
\hline
\end{tabular}

Analysing the experimental data, it is easy to see that a relatively low rate dependency of the Young's modulus has been observed. Comparing to the strength measurements, a larger scatter in the Young's modulus measurements arises from the complexity in extracting this quantity from the SHPB test data. On the other hand, compressive strength of the material shows a clearly increasing trend with the strain rate.

\section{Representation of Strain Rate Sensitivity}

To identify an appropriate method for assigning rate sensitivity to the material properties of the resin, a literature review has been carried out as presented previously. It was concluded that phenomenological and empirical models are often used for this purpose, where the experimental data are fitting to an appropriate function. For the applications in this paper, three such models were considered which suggest logarithmic or exponential dependency of the elastic and strength properties on the strain rate as follows, each involve three material constants to be determined.

$$
\begin{aligned}
\text { Model } 1 \text { [오류! 책갈피가 정의되어 있지 } \\
\text { 않습니다.]: } A_{r t}(\dot{\varepsilon})=A_{0}\left[1+C_{A} \ln \left(\frac{\dot{\varepsilon}}{\dot{\varepsilon}_{0}}\right)\right]
\end{aligned}
$$

Model 2 [오류! 책갈피가 정의되어 있지

$$
\text { 않습니다.]: } \mathrm{A}_{\mathrm{rt}}(\dot{\varepsilon})=\mathrm{A}_{0}\left[1+\left(\frac{\dot{\varepsilon}}{\mathrm{c}}\right)^{\frac{1}{\mathrm{P}}}\right]
$$

$$
\text { Model } 3 \text { [12]: } A_{\text {rt }}(\dot{\varepsilon})=a+b \dot{\varepsilon}^{c}
$$

where, $A_{r t}$ can be the Young's modulus or strength as a function of strain rate, $A_{0}$ is the quasi-static value of the appropriate parameter and $\dot{\varepsilon}$ the current effective strain rate, $\dot{\varepsilon}_{0}$ a reference strain rate. Quantities $C_{A}$ in Eq. (1), $C$ and $P$ in Eq. (2) and $a, b$ and $c$ in Eq. (3) are the parameters of the models, which are determined by fitting the experimental data to the 
respective expressions.

The Matlab curve fitting toolbox was applied to fit the measured elastic and strength properties of the resin with Eqs. (1)-(3). The accuracy of the fitting obtained with each of the models was assessed by calculating the coefficient of determination, $\mathrm{R}^{2}$, and Root Mean Square Error (RMSE). The RMSE allows comparing between the accuracy of the fitting for different models. The lower the RMSE, the more accurate the data fitting to the formula. On the other hand, the $\mathrm{R}^{2}$ provides an absolute measure of the error, where $\mathrm{R}^{2}=1$ indicates a perfect fit, while $\mathrm{R}^{2}$ close to zero suggests a poor fit.

Comparing value of $\mathrm{R}^{2}$ and RMSE presented in Table 2 for the three models, it is easy to see that all the models have a similar accuracy for the Young's modulus while for the strength Models 2 and 3 appear to outperform Model 1, which can be expected as the former ones have more fitting parameters than the last. However, despite providing a better fit within the data range considered, models 2 and 3 suggest an exponential dependency on the strain rate, hence are likely to overestimate the material properties beyond this range, because the exponential growth is rapid. To avoid this, the logarithmic dependence as defined in Eq. (1) was adopted to represent the rate sensitivity of the Young's modulus and strength of the resin, which is a relatively conservative approach in comparison with the other two.

Table 2. Estimated errors in the curve fitting.

\begin{tabular}{ccccccc}
\hline & \multicolumn{2}{c}{ For Young's modulus $E(\mathrm{GPa})$} & \multicolumn{3}{c}{ For compressive strength $S_{c}(\mathrm{MPa})$} \\
\hline & Model 1 & Model 2 & Model 3 & Model 1 & Model 2 & Model 3 \\
$\mathrm{R}^{2}$ & 0.2097 & 0.2695 & 0.2696 & 0.489 & 0.8426 & 0.8438 \\
$\mathrm{RMSE}$ & 0.2974 & 0.2953 & 0.3057 & 34.48 & 19.77 & 20.38 \\
\hline
\end{tabular}

Since the quasi-static testing of resin samples was carried out at a strain rate of $0.0004 \mathrm{~s}^{-1}$, this was defined as the value of a quasi-static reference strain rate, $\dot{\varepsilon}_{0}$. The strain rate scaling constant for Young's modulus and strength, $C_{E}$ and $C_{S}$, respectively, were determined by fitting the experimental data to expression (1).

According to equation (1) the normalized stiffness/strength is linearly dependent on the logarithm of the normalized strain rate. The scaling constants for stiffness and strength can then be defined as the gradients of the linearly fitted lines as shown in Fig. 2. The intersections of fitted linear functions with the ordinate axis were set to 1 , so that at the reference strain rate the models would be capable of representing the quasi-static properties. For stiffness, the models reproduce the linear trend reasonably well. In terms of approximation of the strength properties, the data trend was not captured very well. The data points which were not represented properly by the fitted straight line were obtained at relatively lower strain rates, at which very limited number of tests were conducted. In the present work, the models developed were to be applied to simulate the impacts cases, which generate strain rates beyond this range. Therefore, this discrepancy was not expected to cause any problem. The fitting can be potentially improved by carrying out testing of the resin samples over a larger range of strain rates. However, at present it might not be possible due to limitations of the experimental equipment. 


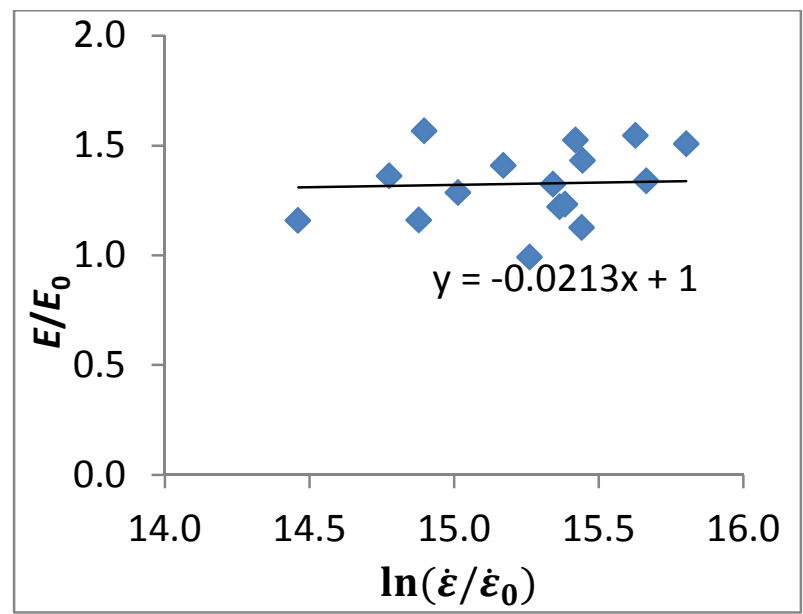

(a) Young's modulus

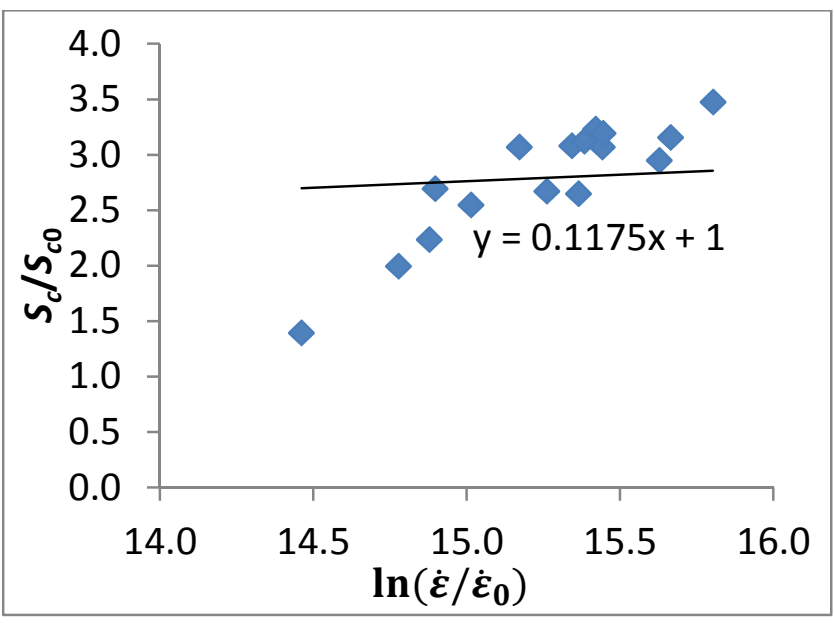

(b) Compressive strength

Fig. 2 Strain rate sensitivity of epoxy resin.

The values of scaling coefficients $C_{E}$ and $C_{S}$ were determined as follows.

$$
C_{E}=0.0213 \quad \text { and } \quad C_{S}=0.1175
$$

Since the resin is an isotropic material, the rate dependence needs to be assigned only to a single elastic property, Young's modulus, $E$. Note that Poisson ratio, $v$, is considered to be unaffected by the strain rate. The rate dependence of the shear modulus, $G$, can be obtained from the isotropy, i.e. $G=E / 2(1+v)$. Failure of the resin was defined by the maximum stress failure criterion, hence the rate dependency was also assigned to tensile and compressive strengths, $S_{t}$ and $S_{c}$. The impact response of the resin under the tension and compression was assumed to be the same, hence the same value of the scaling coefficient, $C_{S}$, was used to prescribe the rate sensitivity to the tensile and compressive strengths.

\section{Conclusion}

To evaluate the strain rate sensitivity of the resin, mechanical properties of the epoxy resin were determined experimentally at the quasi-static and high strain rate loading. The experimental data were approximated by a logarithmic model. In this way, the parameters of the model were determined and they are applied to represent the strain rate sensitivity of epoxy resin.

The testing results indicated that the strain rate sensitivity of compressive strength of epoxy resin is more significantly than that of the Young's modulus.

With rate-dependent properties of the resin known, the rate-dependency of carbon fiber reinforced composite materials can be predicted by applying FEM at micro- and meso- scales unit cell models.

\section{Acknowledgement}

The financial support from Science and Technology Commission of Minhang District, through contract No. 201501-MH-C101-007 is gratefully acknowledged.

\section{References}

[1] W. W. Chen, B. Song, Split Hopkinson (Kolsky) Bar: Design, Testing and Applications, Mechanical Engineering Series, DOI 10.1007/978-1-4419-7982-7_1, 2011

[2] J. Harding, E. O. Wood, J. D. Campbell, Tensile testing of materials at impact rates of strain, J. Mech. Eng. Sci. 2(2) (1960) 88-96. 
[3] W. Hufenbach, A. Langkamp, M. Gude, C. Ebert, A. Hornig, S. Nitschke, H. Böhm, Characterization of strain rate dependent material properties of textile reinforced thermoplastics for crash and impact analysis, Mater. Sci. 2 (2013) 204-211.

[4] C. Ebert, W. Hufenbach, A. Langkamp, M. Gude, Modelling of strain rate dependent deformation behaviour of polypropylene, Polym. Test. 30(2) (2011) 183-187.

[5] D. Mohotti, M. Ali, T. Ngo, J. Lu, P. Mendis, Strain rate dependent constitutive model for predicting the material behaviour of pleurae under high strain rate tensile loading, Mater. Des. 53 (2014) 830-837.

[6] I. Daniel, B. Werner, J. Fenner, Strain-rate-dependent failure criteria for composites, Compos. Sci. Technol. 71(3) (2011) 357-364.

[7] I. Daniel, J. Luo, M. Schubel, B. Werner, Interfibre/interlaminar failure of composites under multi-axial states of stress, Compos. Sci. Technol. 69(6) (2009) 764-771.

[8] G. Cowper, P. Symonds, Strain hardening and strain-rate effects in the impact loading of cantilever beams, Tech. Rep., Brown University Division of Applied Mathematics, 1957.

[9] C. Yen, Ballistic impact modelling of composite materials, 7th international Ls-Dynauser's conference, Dearborn, Michigan, 2002.

[10] M. Lingett, Multi-scale modelling of polymer composite materials at high rate of strain, $\mathrm{PhD}$ thesis, University Of Nottingham, 2012.

[11] W. W. Chen, B. Song, Split Hopkinson (Kolsky) Bar: Design, testing and applications, Mechanical Engineering Series, DOI 10.1007/978-1-4419-7982-7, (C) Springer science+ Business Media, LLC, 2011.

[12] M. Mahmood, J. Majid, Investigation of strain rate effects on in-plane shear properties of glass/epoxy composite, Compos. Struct. 91(1) (2009) 95-102. 